\title{
EFFECT OF GRAVITY ON THE KINETICS OF CONSOLIDATION OF POWDER BODIES
}

\author{
R.M. KADUSHNIKOV, E.Y. NURKANOV*, \\ A.V. SHAK, M.S. PETROV and V.V. KARTASHOV \\ Urals State Technical University, 19 Mira, Ekaterinburg 620002, Russia
}

(Received 26 November 2002)

\begin{abstract}
Physical mathematical model of sintering powders is suggested with respect to the influence of gravitation on sintering kinetics. Structure genesis and evolution in sintered glass-based particulate bodies is investigated both in computer simulations and "in situ".
\end{abstract}

Keywords: Computer simulations; Particulate body; Structure genesis and evolution; Consolidation; Sintering kinetics

\section{INTRODUCTION}

The existing models of sintering powder bodies can be conditionally divided into two categories. The first-category models are referred to a powder body as closed (isolated) systems in which external forces are absent. The models of the second category treat a powder body as nonisolated one that is affected by external bodies, fields, etc. According to the classical models (Frenkel, 1946; Pines, 1946; Kuczynski, 1949), spontaneous densification of a porous body results from either diffusion processes or the action of capillary Laplace forces that lead to deformation of the porous body. To simulate the processes of the structure genesis and evolution in the powder substances sintered, a method of discrete elements is applied, sintering being represented as the motion and interpenetrating of spherical particles. In this case, the motive force of the process is considered to be the excessive surface energy, with the action of the gravity being often disregarded (Kadushnikov et al., 1991a,b; Rodriquez et al., 1985; Gubernat and Rinderle, 1988).

Based on the rheological theory of sintering, the models were developed (Skorokhod, 1972; Olevsky, 1998) that substantiated from the thermodynamical point of view the densification of a porous body as a deformational process and allowed for the external action factor in the approximation of uniaxial compression. However, in the framework

\footnotetext{
*Corresponding author.
} 
of such approach stress anisotropy is ignored that is caused by the gravity and internal capillary forces in the powder body deformed. Another approach was described in Olevsky and German (2000) and Olevsky et al. (2000), where the authors used the conception of a linear distribution by the sample height of internal stresses (due to gravitation), which allowed one to draw the rheological theory of sintering a continuous medium (Olevsky, 1998) in order to determine strains and anisotropic densification in the sample upon shrinkage. Thus, the relation between the shrinkage anisotropy and the gravity upon sintering a powder body was unambiguously established. Yet, the question on the necessity of taking into account the gravity in model approximations for sintering still remains open, as well as the question on the role of gravity in the formation of nonstationary kinetics of shrinkage and consolidation.

The tasks of the work presented are numerical simulation of the gravity action on the sintering of powder bodies and determination of the gravity contribution to the formation of nonstationary kinetics of shrinkage and consolidation in the case of diffusive-viscous mechanism of mass transfer. To solve the problems stated simultaneous calculation and in situ experiments were conducted. Calculation experiments were performed using SIAMS S3D - software based on the structure-simulating model of sintering of spherical particles (Kamenin et al., 1998). In situ experiments consisted in isothermal sintering of the ensembles of spherical particles made of quartz glass.

\section{STRUCTURE-SIMULATING MODEL OF SINTERING A POWDER BODY}

For computations, a structure-simulating model of sintering of spherical particles was used (Kamenin et al., 1998) that treats the process of structure genesis and evolution upon sintering in view of its three-stage representation: filling, sintering, and recrystallization and normal grain growth.

The result of the first stage of filling and formation is reproduced with a random close packing of spherical particles. To construct the close packing of spherical particles in a bunker with flat walls the algorithm "drop and roll" was chosen. The physical idea of this algorithm consists in the following. Spheres are generated according to a given law of size distribution of particles and are dropped into the bunker either to one and the same point or to a randomly chosen position. As the falling sphere encounters an obstacle - bunker wall or already packed sphere - it sticks to it (without hitting) and starts sliding across the surface in the direction of the minimum potential energy (projection on the obstacle surface of the direction of the sphere's free-fall) as far as the following obstacle. The sphere stops evidently in the point of intersection of three surfaces (three spheres, two spheres and a plane, etc.) or on the surface perpendicular to the direction of the sphere's free-fall (for example, on the bunker bottom).

The constructed close packing of spheres represents initial data for simulating the sintering stage (second stage) which is described by the Newton's equations of free motion for spherical particles. When deriving the above equations, the task was formulated to determine, using the known laws of pair interaction of particles upon sintering, the process staging for an unlimited ensemble of spherical particles. Evolving system of $N$ spherical particles $S_{i}\left(R_{i}, \mathbf{r}_{i}, \mathbf{v}_{i}\right)$ is described by the following parameters: $i=1 \ldots N, R_{i}$ is the sphere radius, $\mathbf{r}_{i}$ is the coordinate of the sphere center, $\mathbf{v}_{i}$ is the rate of the sphere motion in the bunker with $K$ flat walls: $\left(\mathbf{r}, \mathbf{b}_{j}\right)=D_{j}, j=1, K$; $\mathbf{b}_{i}$ is the normal to the bunker walls. 
When the contact takes place, one should allow for the interaction of spheres which leads to sintering. The distance between centers of the in-pair touched spheres:

$$
\left|\mathbf{r}_{i j}\right|=\left|\mathbf{r}_{i}-\mathbf{r}_{j}\right|<R_{i}+R_{j}
$$

Equation of the sintering kinetics for all pairs of incident (contacting) spheres can be written in the form:

$$
\mathbf{v}_{i j}=\dot{\mathbf{r}}_{i j}=f\left(\left|\mathbf{r}_{i j}\right|\right) \mathbf{r}_{i j}, \quad i=1, N ; j \rightarrow i
$$

(the last notation means that " $j$ is incident to $i$ "). For condition (1) to be fulfilled for each pair of interacting particles, equations of motion should have the following form:

$$
\left\{\begin{array}{l}
\dot{\mathbf{r}}_{i}=\mathbf{v}_{i}-\mu \sum_{j \rightarrow i}\left(\mathbf{v}_{i j}-f\left(\left|\mathbf{r}_{i j}\right|\right) \mathbf{r}_{i j}\right)\left(f\left(\left|\mathbf{r}_{i j}\right|\right)+f^{\prime}\left(\left|\mathbf{r}_{i j}\right|\right)\left|\mathbf{r}_{i j}\right|\right) \\
\dot{\mathbf{v}}_{i}=\frac{\mathbf{F}_{i}}{m_{i}}-3 \frac{\mathbf{v}_{i}\left|\dot{\mathbf{r}}_{i}\right|}{R_{i}\left|S_{i}\right|} \cdot \Xi_{i}-\mu \sum_{j \rightarrow i}\left(\mathbf{v}_{i j}-f\left(\left|\mathbf{r}_{i j}\right|\right) \mathbf{r}_{i j}\right) \\
\dot{R}_{i}=\frac{\left|\dot{\mathbf{r}}_{i}\right| \cdot \Xi_{i}}{\left|S_{i}\right|}
\end{array}\right.
$$

where $m_{i}=4 / 3 \pi \rho R_{i}^{3}, \rho$ is the density of the sphere material, $\mathbf{F}_{i}$ is the sum of external forces acting on each sphere; $\mu$ is the weight multiple which is actually responsible for the influence of sintering on the motion of system of spheres; $\left|S_{i}\right|=\int_{\Phi_{i}} \int d s=$ $\partial V / \partial R_{i}$ is the free-surface area $\Phi_{i}$ of the $i$ th sphere, $\partial V / \partial r_{i}=\Xi_{i}=\int_{\Psi i} \int\left(\mathbf{r}_{i} /\left|\dot{\mathbf{r}}_{i}\right|\right) \mathbf{n} d s$ can be taken as the area of projection of the occupied surface $\Psi_{i}$ of the $i$ th sphere on the plane normal to $\dot{\mathbf{r}}_{i}$ ( $\mathbf{n}$ is the unit vector of the normal to surface), $V$ is the united volume of the spheres.

The third stage (recrystallization and normal grain growth) is entirely controlled by the motion and sintering of spheres; therefore, the same system of equations (2) is applied. The effects of normal grain growth are taken into account only in the equations of the sphere-size dynamics; namely, for spheres with the surface completely covered by neighbors (not exerting the influence on the mass preservation) equations for the dynamics of normal grain growth are used:

$$
\dot{R}_{i}=\lambda \sum_{j \rightarrow i}\left(\frac{1}{R_{j}}-\frac{1}{R_{i}}\right),
$$

where $R_{i}$ is the radius of the $i$ th particle entirely surrounded by $j$ particles with the radii $R_{j}$.

Thus, at a certain instant $t$ the state of the physical system is described by the set of positions and velocities of particles $\left\{\mathbf{r}_{i}(t), \mathbf{v}_{i}(t), i=1, N\right\}$. When cycling time step, these values are recalculated with the use of interaction forces and equation of motion to obtain the system state at a certain later time instant $t+d t$.

Function $f\left(\left|\mathbf{r}_{i j}\right|\right)$ has the following form: 


$$
\mathbf{v}_{i j}=f\left(\left|\mathbf{r}_{i j}\right|\right) \mathbf{r}_{i j}, \quad f\left(\left|\mathbf{r}_{i j}\right|\right)=\frac{B}{m \varepsilon(1-\varepsilon)^{m-1}}, \quad \varepsilon=\frac{\left|\mathbf{r}_{i j}\right|}{\left|\mathbf{r}_{i j}^{(0)}\right|}
$$

Expression (3) can be derived if to differentiate with respect to time the known phenomenological formula (Geguzin, 1984) that describes the kinetics of the initial stage of mutual sintering of spherical grains

$$
\left(\frac{\left|\mathbf{r}_{i j}^{(0)}-\mathbf{r}_{i j}\right|}{\left|\mathbf{r}_{i j}^{(0)}\right|}\right)^{m}=B t
$$

where $\mathbf{r}_{i j}, \mathbf{r}_{i j}^{(0)}$ are the current and the initial distance between the centers of contacting particles; $B$ and $m$ are the constants defined by the type of substance and the mechanism of mass transfer. For the mechanism of diffusive-viscous flow

$$
m=1, \quad B=\frac{\lambda}{\left|\mathbf{r}_{i j}^{(0)}\right|}, \quad \lambda=\frac{3 \sigma}{4 \eta}
$$

where $\eta$ is the viscosity coefficient, $\sigma$ is the specific surface energy.

The $\eta$ and $\sigma$ values can be different from those for real particulate bodies by several orders, depending on the sintering temperature, crystallinity, etc, which, with the substitution of the material constants into the system of equations (2) leads to an essential scatter of times of integrating the above system of equations. To numerically investigate sintering of powder substances with different $\eta$ and $\sigma$ on the unified time scale, the physical scaling of the duration of integrating the system of equations (2) is required. For this purpose, let us introduce the conception of equivalent states in terms of energy for systems of spherical particles under sintering. The systems of sintered spherical particles with the same initial configuration of particles and different viscosity and surface energy have energetically equivalent states provided that internal Laplace forces perform equal work on shifting particles.

Treat two systems of spherical particles with the same initial configuration and characteristics $[\sigma, \lambda]$ and $\left[\sigma^{\prime}, \lambda^{\prime}\right]$, in so doing, $\lambda^{\prime}=\omega \lambda, \lambda=3 \sigma / 4 \eta$. The work on shifting the $i$ th particle for the time $\Delta \tau$ equals

$$
\Delta \mathrm{W}_{i}=\mathrm{v}_{i} F_{\mathrm{L}} \Delta \tau
$$

where $\mathrm{v}_{i}$ is the rate of $i$ th particle, $F_{\mathrm{L}}$ is the pressing force in the zone of contacting two particles. According to Balshin (1972) for $F_{\mathrm{L}}$ the following is valid

$$
F_{\mathrm{L}}=2 \pi \sigma R
$$

thereto, formula (4) is true up to $X \leq 0.5 R$ ( $X$ is the contact chord; $R$ is the radius of a particle), and the condition for the work equality in two systems under consideration is expressed as follows: 


$$
\begin{aligned}
& \Delta \mathrm{W}_{i}=\Delta \mathrm{W}_{i}^{\prime} \\
& \sigma \mathrm{v}_{i} \Delta \tau=\sigma^{\prime} \mathrm{v}_{i}^{\prime} \Delta \tau^{\prime}
\end{aligned}
$$

For the system of equations (2) one can prove that in case $F_{i}=0, \lambda^{\prime}=\omega \lambda,(\lambda=3 \sigma / 4 \eta)$ the following equality is fulfilled:

$$
\mathrm{v}_{i}^{\prime}=\omega \mathrm{v}_{i}
$$

Substituting (6) into (5) we have

$$
\sigma \lambda \Delta \tau=\sigma^{\prime} \lambda_{i}^{\prime} \Delta \tau^{\prime}
$$

Provided that condition (7) is satisfied, the system states with characteristics $[\sigma, \lambda]$ and $\left[\sigma^{\prime}, \lambda^{\prime}\right]$ can be considered to be energetically equivalent and the quantity $\Omega=\sigma \lambda \Delta \tau=\sigma^{\prime} \lambda^{\prime} \Delta \tau^{\prime}$ to be the measure of duration of integrating system of equations (2) or the measure of duration of evolution for a system of spherical particles in computations. The parameter $\lambda$ will be called conditional rate of sintering.

It should be noted that for system of equations (2) in the gravitation field $\left(F_{i} \neq 0\right)$ condition (7) is not fulfilled, therefore, the evolution duration for the system of spherical particles in this case is measured in absolute time units $\tau$.

\section{COMPUTATION ROUTINE}

All computations are conducted based on the above-described structure-imitating model of sintering particulate body. In all computational experiments a standard initial packing of spherical particles (in what follows, package) with equal characteristics is employed: the particle number $N=5000$, the particle radius $R=1$. Closely packed structure of spherical particles with the given characteristics (Fig. 1) was constructed in a bunker with square base and linear dimensions $h$, the height, and $d$, the base side $(h / d=3)$.

To determine the contribution from the gravity and internal Laplace forces to the kinetics of sintering the package, computations are carried out separately for the cases $F_{i}=0$ and $F_{i}=m g$, where $F_{i}$ is the external force (in particular, gravity). Upon simulating in the gravitation field, the acceleration of free fall is set equal to $g=10$ $\left[\mathrm{m} / \mathrm{sec}^{2}\right]$. Sintering in both cases is considered to be isothermal and governed by the mechanism viscous flow of the substance. Numerical calculations of system

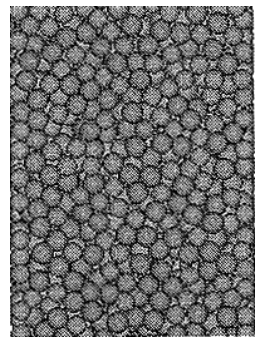

FIGURE 1 Fragment of initial model close packing of spherical particles. 
of equations (2) is performed for the values of the conditional rate of sintering $\lambda=10^{-1}$, $10^{-2}, 10^{-3}$. Deformation is estimated by the relative linear shrinkage of the package $L_{\mathrm{s}}$, consolidation, by the parameter of $\alpha$. The parameters $\alpha$ and $L_{\mathrm{s}}$ are calculated as follows:

$$
L_{\mathrm{s}}=\left(h_{0}-h\right) / h_{0}, \quad \alpha=\left(P-P_{0}\right) /\left(1-P_{0}\right),
$$

where $h$ is the current height of package; $h_{0}$ is the initial package height; $P$ is the current relative density; $P_{0}$ is the initial relative density.

For estimation of the character of density distribution along the package height the parameter $\rho$ is employed, which is calculated as follows:

$$
\rho=\frac{\sum_{k} V_{k}(z)}{h S_{\mathrm{b}}}
$$

where $V_{k}(z)$ is the volume of a sphere's layer of the $k$ th particle enclosed between the planes $z=z_{i}$ and $z=z_{i}+h ; i=H / h, H$ is the package height, $h$ is the layer thickness, $S_{\mathrm{b}}$ is the area of the bunker's base.

It should be noted that the parameter $\rho$ defines the local relative density and the degree of interpenetration of particles; $\rho$ takes up values $\rho>1$ in the case when in the package layer considered, there takes place a significant interpenetration of particles.

\section{REGULARITIES OF SINTERING KINETICS IN "WEIGHTLESSNESS" (COMPUTATIONS)}

Computations on sintering under condition $F_{i}=0\left(F_{i}\right.$ is the external force) allowed us to establish a number of important regularities. As turned out, the kinetics of shrinkage and consolidation of the package at $\Omega<0.6$ exhibited virtually linear behavior for the considered values $\lambda$ (Fig. 2). At values higher than $\Omega=0.6$ nonstationary kinetics of shrinkage and consolidation manifests itself in a nonlinear dependence $L_{\mathrm{s}}$ and $\alpha$ on $\Omega$ (Fig. 2), with the shrinkage parameter $\alpha$ clearly pointing to the deceleration of consolidation.

The linear kinetics of shrinkage and consolidation at the initial stage can be expected, since for the mechanism of viscous flow of substance, the velocity of approaching the centers of contacting particles is virtually constant in the isothermal conditions $(\lambda=3 \sigma / 4)$. In other words, the isothermal sintering of the spherical-shape particles is described by the law of constant velocity of the substance flow $(\Delta V / \Delta t=$ const, $V$ is the particle volume) which is valid both for pure viscous flow and for a combination of plastic and viscous flows (Balshin, 1972). Appearance of the nonlinear portion in the curve $\alpha(\Omega)$ (Fig. 2) is most probably related to the effect of zonal isolation (Balshin, 1972; Skorokhod and Solonin, 1984) that will be treated in more detail for the case of sintering in the gravitation field.

The effect of zonal isolation can be in part diminished by governing $\lambda$. Thus, at the same other conditions, a decrease of $\lambda$ caused the rise of the curves $L_{\mathrm{s}}(\Omega)$ and $\alpha(\Omega)$ (Fig. 2 ), which means an enhancement of the sintering coherency. Such a behavior of the system can be interpreted as follows. While contacting two balls in the neck of contact 


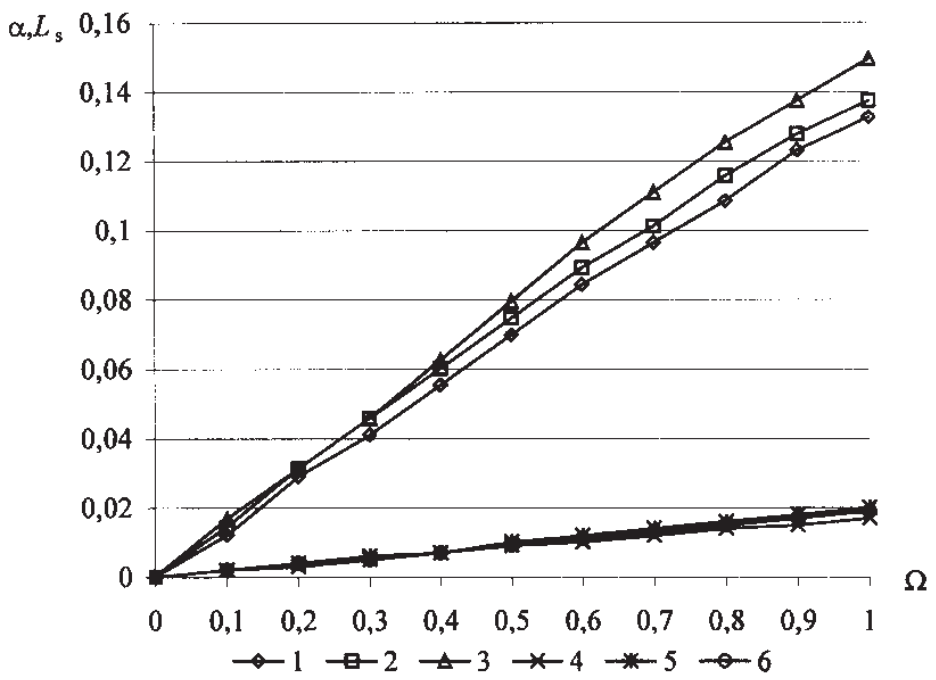

FIGURE 2 Dependencies of the parameter of consolidation $\alpha$ and relative shrinkage $L_{\mathrm{s}}$ on $\Omega$ for a model package of spherical particles obtained in computations at $F_{i}=0\left(F_{i}\right.$ is the external force, in particular, gravity); $\alpha(\Omega)$ upon sintering with $(1) \lambda=10^{-1} ;(2) \lambda=10^{-2} ;(3) \lambda=10^{-3} ; L_{\mathrm{s}}(\Omega)$ upon sintering with (4) $\lambda=10^{-1}$; (5) $\lambda=10^{-2}$; (6) $\lambda=10^{-3}$.

there arises a contact stress $p_{k}$. In flowing by-contact areas in any direction, the contact stress $p_{k}=$ const (Balshin, 1972). Therefore, the contact pressing stress $p_{k}$, that is normal to the contact area $A=\pi x^{2}$ ( $x$ is the radius of the contact circle) can be written as

$$
p_{k}=2 R \sigma / x^{2}
$$

where $R$ is the radius of a particle; $\sigma$ is the surface tension.

The radius of contact $x$ can be expressed through $L$, the distance between the centers of contacting particles (Geguzin, 1984)

$$
\frac{\Delta L}{L_{0}} \approx \frac{x^{2}}{4 R^{2}}, \quad \Delta L=L_{0}-L, \quad L_{0}=2 R,
$$

where $L_{0}$ is the initial distance between the centers of contacting particles. Substituting (9) into (8) we have

$$
p_{k} \approx \sigma / \Delta L, \quad p_{k} \approx \sigma / \lambda \Delta t
$$

where $\lambda$ is the conditional rate of sintering and at the same time rate of approaching the centers of the contacting particles.

From (10) it follows that the lower the $\lambda$ value, the more stressed the initial state of the system is. Thus, treating the system sintering as the deformational process, we can apparently consider a greater shrinkage and consolidation as a consequence of a more stressed initial state. 


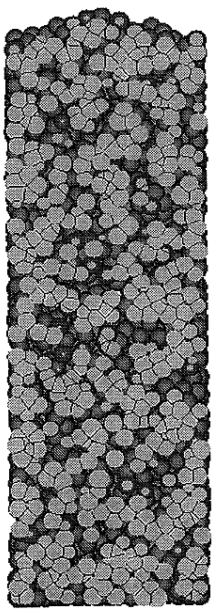

1

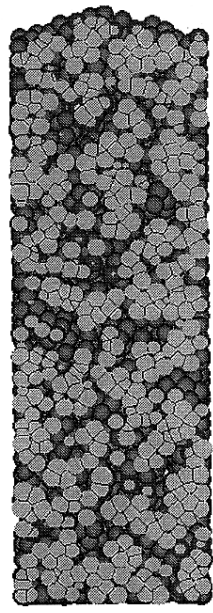

2

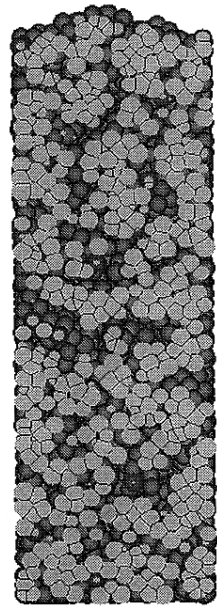

3

FIGURE 3 Microstructure of a model package of spherical particles in simulation of sintering by the mechanism of viscous flow. Conditions of computations: $F_{i}=0$ (external force, in the given case, gravity); duration of sintering in computations $\Omega=1$; (1) $\lambda=10^{-1}$; (2) $\lambda=10^{-2}$; (3) $\lambda=10^{-3}$.

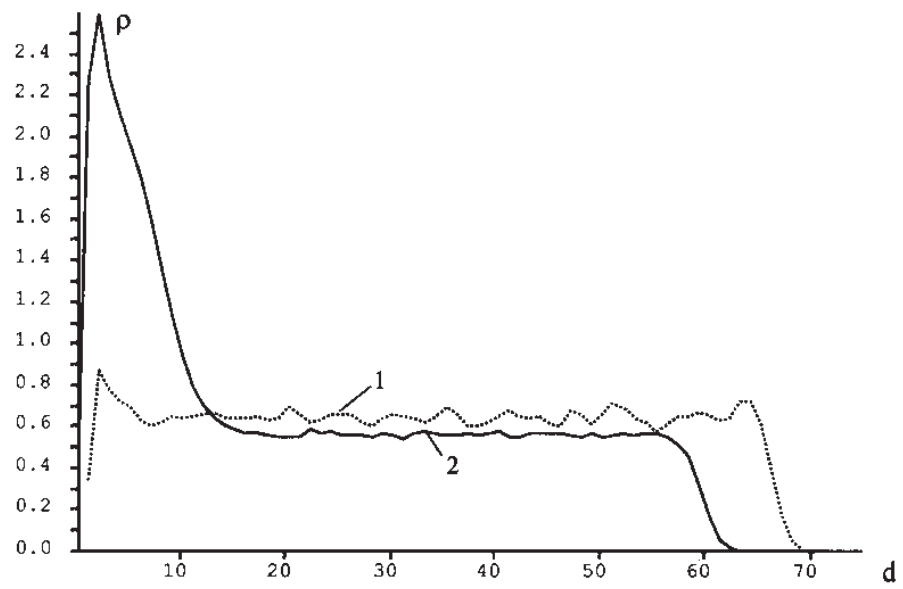

FIGURE 4 Distribution $\rho$ along the height $h$ in model packages of spherical particles in simulation of sintering by the mechanism of viscous flow and with the conditions of sintering in computations: (1) $\Omega=1$, $F_{i}=0, \lambda=10^{-2}$; (2) $\tau=5 \mathrm{~h}, F_{i}=m g, g=10, \lambda=10^{-2} ; \rho$ is the local density and the degree of interpenetration of particles.

A characteristic sign of sintering for the case $F_{i}=0$ was the formation of pores and porous channels (Fig. 3) which were quite uniformly distributed along the height of a package, which is evidenced by the uniform dependence $\rho(h)$ for the central part of the package (Fig. 4). In the bottom part of the package $\rho$ has an increased value, which testifies to the formation of the bottom "skin", that is, a compacted zone with a significant degree of interpenetration of particles. 


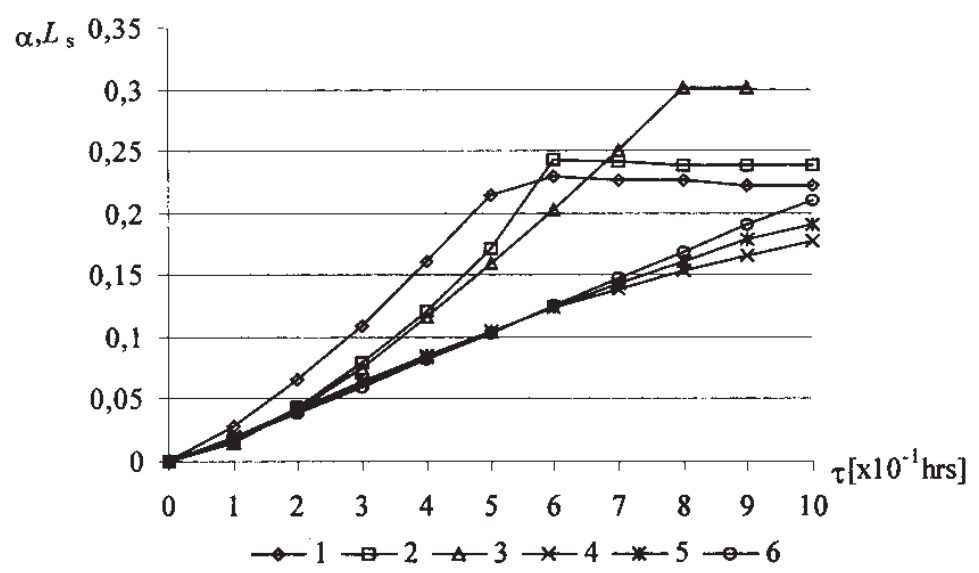

FIGURE 5 Time dependencies of the parameter of consolidation $\alpha$ and relative linear shrinkage $L_{\mathrm{s}}$ of a model package of spherical particles obtained in computations at $F_{i}=m g, g=10$ ( $F_{i}$ is the gravity): $\alpha(\tau)$ upon sintering with $(1) \lambda=10^{-1} ;(2) \lambda=10^{-2} ;(3) \lambda=10^{-3} ; L_{\mathrm{s}}(\tau)$ upon sintering with $(4) \lambda=10^{-1} ;(5) \lambda=10^{-2}$; (6) $\lambda=10^{-3}$.

\section{FORMATION OF NONSTATIONARY KINETICS OF SINTERING IN THE GRAVITATION FIELD (COMPUTATIONS)}

Computations on simulating the sintering in the gravitation field $\left(F_{i}=m g, g=10\right)$ showed that the gravitation field essentially modified the sintering mode and the kinetic characteristics of sintering in comparison with the case $F_{i}=0$. Thus, allowance for the gravity resulted in the changes in time scale of the processes considered. For the case $F_{i}=0$ it was necessary to reduce the duration of sintering for systems with different $\eta$ and $\sigma$ to the unified time scale $\Omega$. Application of the gravity resulted in that the sintering of systems with different material constants $\eta$ and $\sigma$ took place in the limits $\tau=10$, where $\tau$ is the time of integrating the system of equations (2). The time of sintering $\tau=10$ was fixed by the leveling-out the curves of consolidation $\alpha(\tau)$ (Fig. 5).

Nonstationary kinetics of consolidation in the case $\left(F_{i}=m g, g=10\right)$ manifested itself already at early stages of sintering and had quite complicated character. Initially, a certain acceleration of the total consolidation of the package (Fig. 5) was observed, which then changed to an abrupt slowing-down up to absolute completion at late stages of sintering. The above circumstance is likely to be considered in the framework of theory of zonal isolation and the conception of mesostructure. According to Balshin (1972), sintering without an external pressure, as a rule, results in a spontaneous agglomeration of particles in groups, that is, shrinkage or contraction of the particles takes place in the boundaries of multiple separate zones. The above phenomenon is conditionally called zonal isolation. According to Nikolenko (1995), zonal segregation is predefined by structure of mesoelements or mesostructure which is inherited upon consolidation. Mesoelement is a region of package that consists of particles with a higher coordination number and in which boundaries the contraction of particles to a cluster occurs. However, with application on the system of gravitation conditions, the initial structure of mesoelements did not exhibit an ability to be inherited upon sintering. Computations on sintering simulation in the gravity field showed that in the bottom part of the package, particles grow faster and the degree of interpenetration 


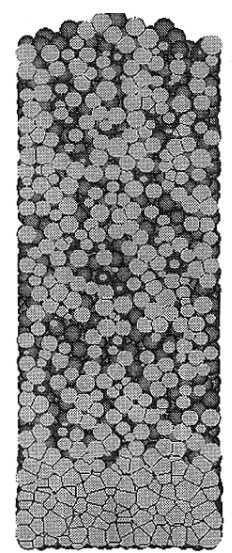

1

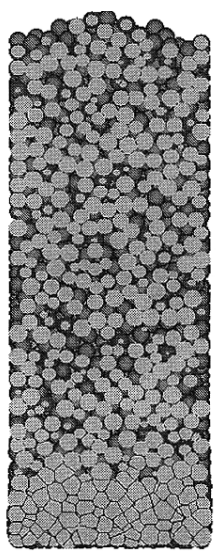

2

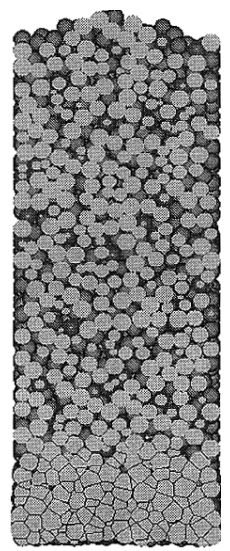

3

FIGURE 6 Microstructure of a model package of spherical particles in simulation of sintering by the mechanism of viscous flow. Conditions of computations: $F_{i}=m g, g=10$ ( $F_{i}$ is the gravity); duration of sintering in computations $\tau=5 \mathrm{~h}$; (1) $\lambda=10^{-1}$; (2) $\lambda=10^{-2}$; (3) $\lambda=10^{-3}$.

of particles is higher than that averaged over volume (Figs. 4 and 6). In the bottom part of the package a cluster is formed that gets enlarged via taking up small clusters located above it. Thus, the total number of clusters (mesoelements) decreases, the zonal isolation is weakened and the homogeneity of sintering grows. Correspondingly, as the sintering homogeneity is a function of the total number of particles, the consolidation (Fig. 5) would accelerate as long as the taking-up of small particles by coarse ones proceeds.

Thus, in computations for the case $F_{i}=m g, g=10$, it was noted that a decrease in $\lambda$ results in a decrease in the consolidation rate (Fig. 5) and an increase in the sintering coherency. The increase in the sintering coherency is evidenced by the highest values of the linear shrinkage and overall consolidation of a package for the conditional rate $\lambda=10^{-3}$ (Fig. 5).

\section{SINTERING OF REAL GLASS-BASED PARTICULATE SYSTEMS}

The mechanism of diffusion-viscous transfer of a substance well agrees with the behavior of glass particles upon sintering, therefore, for the in situ experiments, an ensemble of spherical particles of quartz glass with a size of particles $R \approx 10^{-3} \mathrm{~m}$ ( $R$ is the radius) was chosen. Molding compositions were produced by arbitrary filling of particles in a crucible of cylindrical shape with a height $\mathrm{h} \approx 2.38 \times 10^{-2} \mathrm{~m}$ and diameter $d=1.4 \times 10^{-2} \mathrm{~m}$. For experiments, crucibles of inert ceramic materials (beryllium oxide) were used, which excluded sticking samples to the inner surface of the crucible.

Molding compositions were sintered in the muffle furnace in air at a temperature of $T=950^{\circ} \mathrm{C}$ that is close to the temperature of the onset of softening quartz glass. The sintering temperature was chosen according to literature data (Geguzin, 1984) and the results of preliminary experiments, which showed that at $T=950^{\circ} \mathrm{C}$, sintering becomes apparent already at small exposures. 
The main controlling parameters were $L_{\mathrm{s}}$ relative linear shrinkage of a compact, and $\alpha$, the parameter of consolidation. To determine $\alpha$ the following techniques were employed. The samples sintered were subject to preliminary soaking in nonfoaming epoxy resin. Compacts were cut both longitudinally and transversely; the sections were ground and polished. Images of metallographic sections taken on a light microscope Neophot 32 were analyzed with the use of program-analyzer of images SIAMS600. The relative density $P$ was determined with the use of the above techniques as the volume fraction of particles on a section over the series of metallographic sections.

Analysis of the in situ experimental results showed that upon isothermal free sintering $\left(T=950^{\circ} \mathrm{C}\right.$ ) of the molding compositions of spherical particles made of quartz glass $\left(R \approx 10^{-3} \mathrm{~m}\right)$, there occur the processes of structure formation and consolidation that possess the following features:

(a) spontaneous shrinkage and consolidation take place in the conditions of intense zonal isolation;

(b) zonal isolation results in accumulation of geometrical imperfectnesses which do not relax even after long-term heating of a compact;

(c) the shape of curves of shrinkage and consolidation (Fig. 8) points to a nonstationary kinetics of sintering, with the rate of shrinkage and consolidation being increased at early stages of sintering, which is unexpected because the theory of sintering suggests the kinetics of this phenomenon be monotonously descending (Geguzin, 1984);

(d) maximum intensity of topological transformations occurs during the first 30 minutes of sintering;

(e) average relative density amounts to $P_{0} \approx 0.570$ for a raw molding composition; $P \approx 0.835$ for the compact sintered;

(f) average linear shrinkage of compacts makes up $\approx 20 \%$.

\section{COMPARISON OF THE COMPUTATIONAL AND IN SITU EXPERIMENTAL RESULTS FOR SINTERING SPHERICAL GLASS PARTICLES}

In first turn, one should note close density characteristics of the molding compositions of spherical quartz particles $\left(P_{0}=0.585\right)$ and of the initial model package of spherical particles in computational experiments $\left(P_{0}=0.570\right)$. Thus, a conclusion can be made that the initial level of the structure-geometrical imperfectnesses was similar in the "in situ" and computational models. In situ experiments, on the whole, showed a nonstationary kinetics of shrinkage and consolidation upon isothermal sintering. Close-inshape curves of consolidation $\alpha(t)$, obtained in the in situ experiments (Fig. 8) and in computations on sintering in the gravitation field (Fig. 5) should be noted. The early stage of sintering was characterised by a low rate of consolidation; then, it was succeeded by the stage of accelerated consolidation, and, at last, at further stages, there took place deceleration of consolidation up to reaching a stationary mode (Figs. 5 and 8).

The monotonous kinetics of consolidation shown in works (Geguzin, 1984; Boccaccini and Olevsky, 1999) is in part connected with a size effect. This effect is implied to be the following. For the experiments on sintering the powder of alumino- 
silicate glass BMAS $\left(\mathrm{SiO}_{2}: 48.07 \%, \mathrm{Al}_{2} \mathrm{O}_{3}: 31.64 \%, \mathrm{MgO}: 6.03 \%, \mathrm{BaO}: 13,58 \%\right.$, $T=950^{\circ} \mathrm{C}$ ) (Boccaccini and Olevsky, 1999) the powder was used with the grain size of $10^{-6} \mathrm{~m}$. For the particles of this size, the balance of Laplace capillary forces and the gravity is another than for the particles of quartz glass with a size of $10^{-3} \mathrm{~m}$. The pressing force $F_{\mathrm{L}}$, generated by the Laplace capillary forces, can be estimated by formula (4), and the gravity acting on a particle, by the formula:

$$
F_{\mathrm{g}}=(4 / 3) \pi R^{3} \gamma g
$$

where $\gamma$ is the theoretical density of the particle material. For $R=10^{-6} \mathrm{~m}$, assuming for glass $\sigma=310 \mathrm{mN} / \mathrm{m}$ and $\gamma=2.74 \mathrm{~g} / \mathrm{cm}^{3}$, we obtain $F_{\mathrm{L}}=1.9 \times 10^{-5} \mathrm{~N}$ and $F_{\mathrm{g}}=1.2 \times 10^{-10} \mathrm{~N}$, and for $R=10^{-3} \mathrm{~m}, F_{\mathrm{L}}=1.9 \times 10^{-3} \mathrm{~N}$ and $F_{\mathrm{g}}=1.2 \times 10^{-4} \mathrm{~N}$, respectively. Thus for particles $10^{-3} \mathrm{~m}$ in size $F_{\mathrm{L}} / F_{\mathrm{g}}=16$ and for $10^{-6} \mathrm{~m} F_{\mathrm{L}} /$ $F_{\mathrm{g}}=1.6 \times 10^{5}\left(F_{\mathrm{L}} \gg F_{g}\right)$.

Computations were conducted in our work for two cases: $F_{i}=0$ and $F_{i}=m g, g=10$, where $F_{i}$ is the external force (say, gravity). The condition $F_{i}=0$ in computations is equivalent to the condition $F_{\mathrm{L}} \gg F_{\mathrm{g}}$ in the in situ experiments on sintering powders BMAS with the average particle size of $10^{-6} \mathrm{~m}$ (Boccaccini and Olevsky, 1999). In this case, the dominant part in the formation of the consolidation kinetics is taken by the Laplace capillary forces that provide the monotonous kinetics close to a linear at early stages of sintering (Figs. 2 and 9). Thus, a conclusion can be made that the monotonous kinetics of consolidation without the acceleration stage is observed when the force balance is disturbed in favor of the Laplace capillary forces.

The condition $F_{i}=m g, g=10$ in the computations is equivalent to the condition $F_{\mathrm{L}} /$ $F_{\mathrm{g}}=16$ in the in situ experiments on sintering nonpressed compositions of spherical quartz-glass particles with the average grain size $10^{-3} \mathrm{~m}$ (Figs. 7 and 8 ). In this case, the kinetics of shrinkage and consolidation is affected by the combined action of the gravity and Laplace capillary forces. Thus, one can conclude that the nonstationary kinetics of sintering with the acceleration stage manifests itself in the gravitation field in the conditions when the gravity and Laplace capillary forces turn out comparable by the order of magnitude.

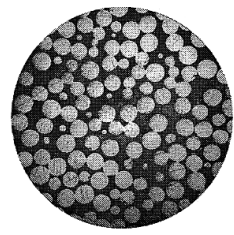

1

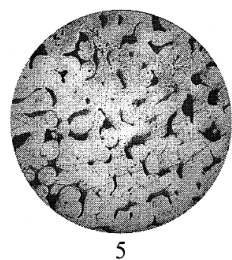

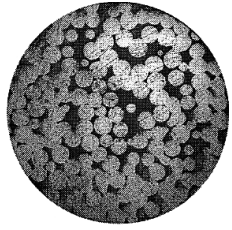

2

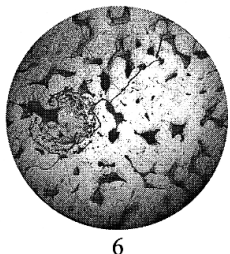

6

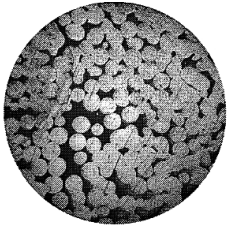

3

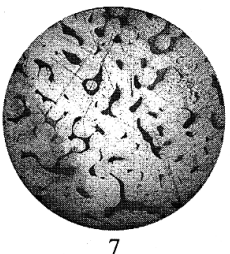

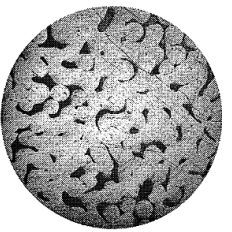

4

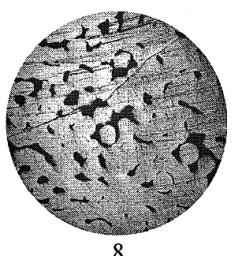

8

FIGURE 7 Structure of moulding compositions of quartz glass with the particle size $R=10^{-3} \mathrm{~m}$ upon free solid-phase sintering $\left(T=950^{\circ} \mathrm{C}\right)$. Duration of sintering (1) $0.25 \mathrm{~h}$; (2) $0.5 \mathrm{~h}$; (3) $0.75 \mathrm{~h}$; (4) $1.0 \mathrm{~h}$; (5) $1.25 \mathrm{~h} ;(6) 1.5 \mathrm{~h}$; (7) $1.75 \mathrm{~h}$; (8) $2 \mathrm{~h}$. 


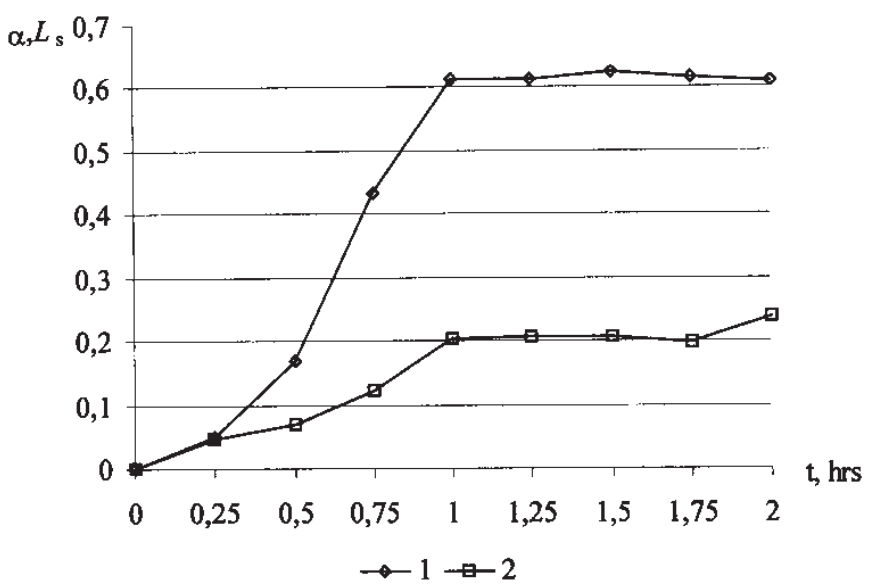

FIGURE 8 Time dependencies of the parameter of consolidation $\alpha$ and relative linear shrinkage $L_{\mathrm{s}}$ of the molding compositions of spherical particles made of quartz glass with the particle size $R \approx 10^{-3} \mathrm{~m}$ upon free solid-phase sintering $\left(T=950^{\circ} \mathrm{C}\right)$ : (1) $\alpha(t)$; (2) $L_{\mathrm{s}}(t)$.

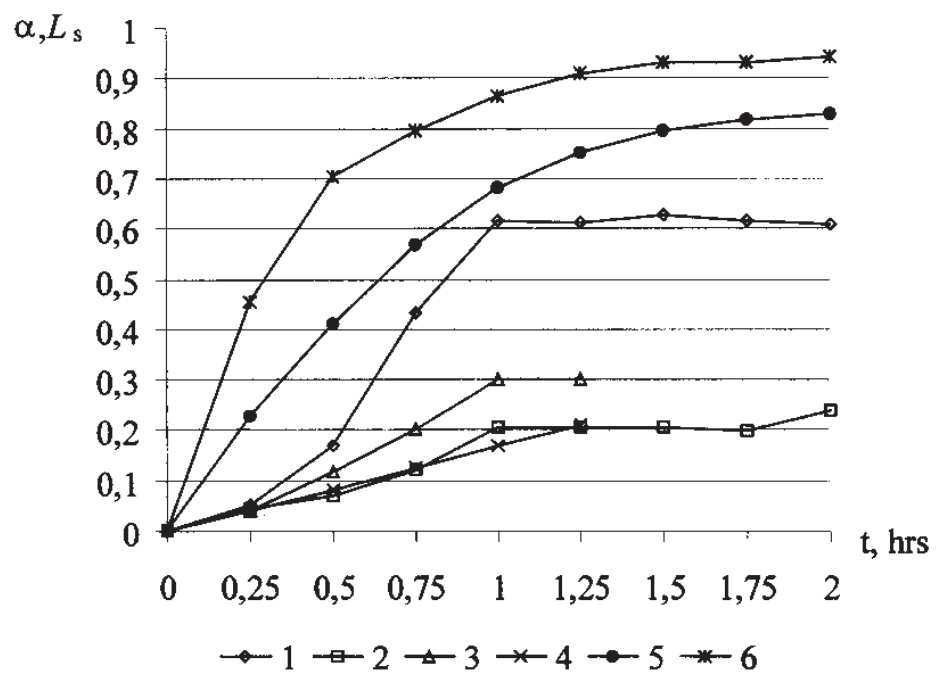

FIGURE 9 Time dependencies of the parameter of consolidation $\alpha$ and relative linear shrinkage $L_{\mathrm{s}}$. In situ experiments: sintering of nonpressed compositions of spherical particles made of quartz glass, average particle radius $R=10^{-3} \mathrm{~m}, T=950^{\circ} \mathrm{C}$, (1) $\alpha(t)$; (2) $L_{\mathrm{s}}(t)$; (5) ordinary form of the consolidation curve $\alpha(t)$ for pressed powders of amorphous materials; (6) $\alpha(t)$ when sintering the pressed powder of alumino-silicate glass BMAS, average particle size $R=10^{-5} \mathrm{~m}, T=950^{\circ} \mathrm{C}$. Computations (3) $\alpha(t)$, (4) $L_{\mathrm{s}}(t)$ upon sintering close packing of spherical particles by the mechanism of viscous flow; the particle size $R=10^{-3} \mathrm{~m}$, conditional rate of sintering $\lambda=10^{-3}$, the gravity $F_{i}=m g, g=10$.

Of special interest is, in the author's opinion, the stage of consolidation acceleration, as in the dilatometrical curves of shrinkage of a conditional form, it is absent, as a rule. In Geguzin (1984), in particular, a general form of the dilatometrical curves of shrinkage of amorphous porous structures is presented (Fig. 9). It is seen that the velocity of consolidation decreases monotonously in the course of sintering. Experiments on sintering the powder BMAS $\left(\mathrm{SiO}_{2}: 48.07 \%, \mathrm{Al}_{2} \mathrm{O}_{3}: 31.64 \%, \mathrm{MgO}: 6.03 \%, \mathrm{BaO}\right.$ : 
$13.58 \%, T=950^{\circ} \mathrm{C}$ ) (Boccaccini and Olevsky, 1999) exhibited the same tendency (Fig. 9), though at early stage, a weak acceleration of consolidation was still observed. Yet, the acceleration stage of consolidation was detected in the kinetic curves $\alpha(t)$ for the sintering of moulding compositions of spherical particles $\left(R=10^{-3} \mathrm{~m}\right)$ (Fig. 8) and in computations (Fig. 5). Since, in both cases, there took place zonal isolation (Figs. 6 and 7), an assumption can be made that the stage of accelerated consolidation is controlled by the effect of "weakening" the zonal isolation in the field of an external force (the gravitation field). Thus, nonstationary kinetics of consolidation is related to a nonlinear decreasing of the total number of clusters in the gravitation field via the taking-up of small clusters by coarser ones.

\section{References}

Balshin, M.Yu. (1972). Scientific Basis of the Powder Metallurgy and Metallurgy of Fibres. Metallurgiya, Moscow.

Boccaccini, A.D. and Olevsky, E.A. (1999). Processing of platelet-reinforced glass-matrix composites: effect of inclusions on sintering anisotropy. Journal of Material Processing Technology, 96, 92-101.

Frenkel, Ya.I. (1946). About viscous flow of solids. Zhournal Eksperimentalnoi Teoreticheskoi Fiziki, 16, 2942.

Geguzin, Ya.E. (1984). Physics of Sintering. Nauka, Moscow.

Gubernat, R. and Rinderle, J.R. (1988). An energy based approach toward determining part shape change during the sintering of ceramic parts. Journal of Engineering Materials and Technology, 110, 305-312.

Kadushnikov, R.M., Alievskii, D.M. and Alievskii, V.M. (1991a). Computer-assisted simulation of the microstructure evolution of polydisperse materials upon sintering. I. Basic terms. Poroshkovaya Metallurgiya, 2, 18-24.

Kadushnikov, R.M., Alievskii, D.M. and Alievskii, V.M. (1991b). Computer-assisted simulation of the microstructure evolution of polidisperse materials upon sintering. II. Zonal isolation. Poroshkovaya Metallurgiya, 5, 5-10.

Kamenin, I.G., Kadushnikov, R.M., Alievsky, V.M., Alievsky, D.M. and Somina, S.V. (1998). 3-Dimensional structure-imitation model of evolution of microstructure of powder body during sintering. Textures and Microstructures, 32, 221-233.

Kuczynski, G.C. (1949). Physics of sintering. Journal of Applied Physics, 20, 1160-1174.

Nikolenko, A.N. (1995), Mesostructure of powder materials. Poroshkovaya Metallurgiya, 11/12, 88-94.

Olevsky, E.A. (1998). Theory of sintering: from discrete to continuum. Materials Science and Engineering, R23, 41-100.

Olevsky, E.A. and German, R.M. (2000). Effect of gravity on dimensional change during sintering - I. Shrinkage anisotropy. Acta Materialia, 48, 1153-1166.

Olevsky, E.A., German, R.M. and Upadhyaya, A. (2000). Effect of gravity on dimensional change during sintering-II. Shape Distortion. Acta Materialia, 48, 1167-1180.

Pines, B.Ya. (1946). About sintering in solid phase. Zhournal Eksperimentalnoi Teoreticheskoi Fiziki, 16, $137-$ 153.

Rodriquez, J., Allibert, C., Mermet, J. and Chaix, J.M. (1985). Simulation de la microstructure au cours du frittage avec phase liquide. Memoireset Etudes Scientifiques Reviue de Metallurgie, 6, 289-296.

Skorokhod, V.V. (1972). Rheological Basis for the Theory of Sintering. Naukova Dumka, Kiev.

Skorokhod, V.V. and Solonin, S.M. (1984). Physico-metallurgical Grounds of Sintering Powders. Metalurgiya, Moscow. 